\title{
Sarin Decomposition on Pristine and Hydroxylated ZnO: Quantum-Chemical Modeling
}

Roman Tsyshevsky1, Scott Holdren², Bryan W. Eichhorn², Michael R. Zachariah³, Maija M. Kuklja $^{1 *}$

${ }^{1}$ Materials Science and Engineering Department, University of Maryland, College Park, MD 20742

${ }^{2}$ Department of Chemistry and Biochemistry, University of Maryland, College Park, MD 20742

${ }^{3}$ Department of Chemical and Environmental Engineering, University of California, Riverside, CA, 92521

\section{Supporting Information}
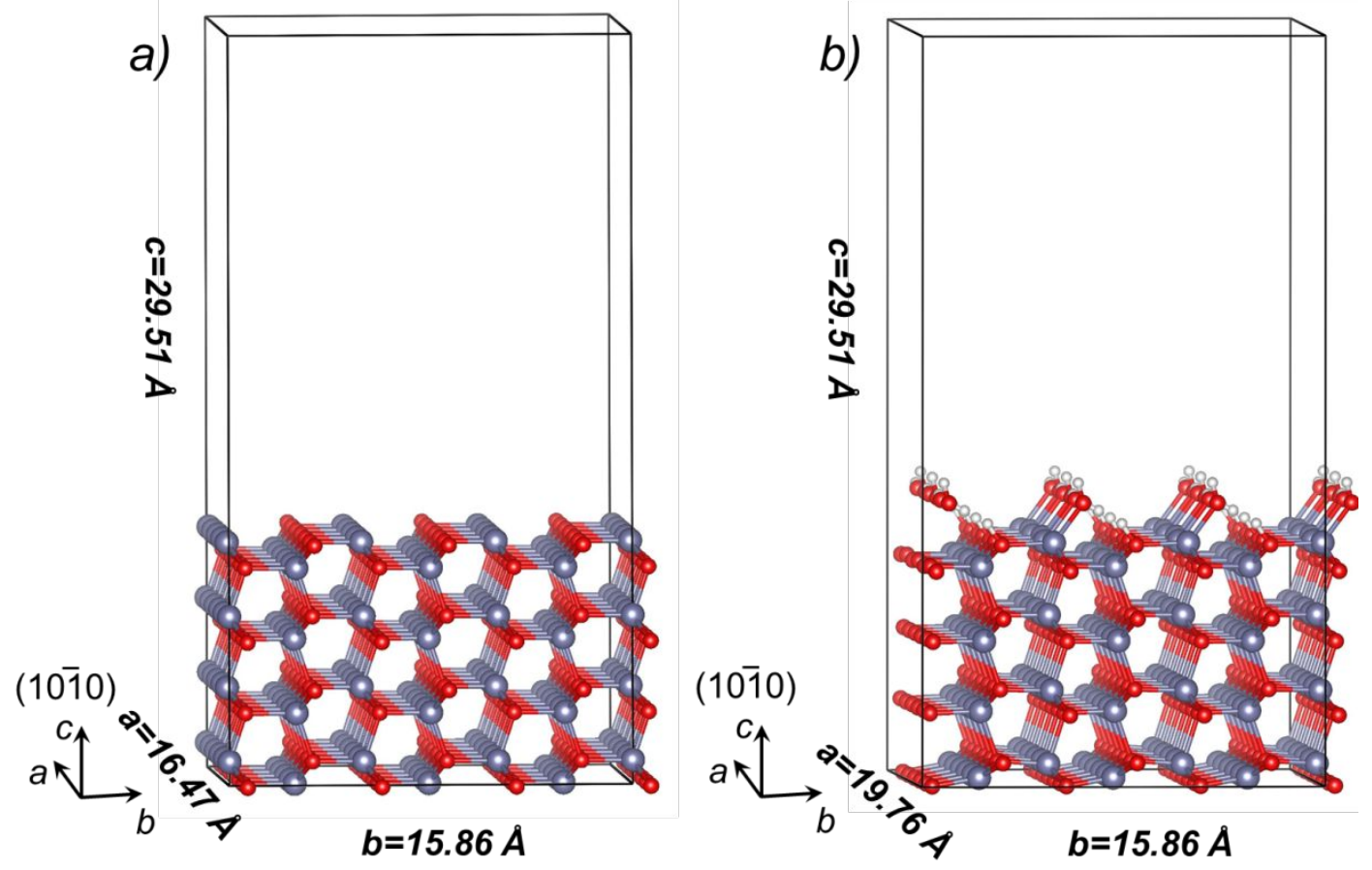

Figure S1. Structures of a) pristine $\mathrm{ZnO}(10 \overline{1} 0)$ surface and b) hydroxylated $\mathrm{ZnO}(10 \overline{1} 0)$ surface with $(2 \times 1)$ water adlayer containing a mix of both intact and dissociated water molecules 


\section{Mechanisms of sarin decomposition on a pristine $\mathrm{ZnO}(10 \overline{10})$ surface (results obtained using PBE functional)}

A schematic representation of the potential energy surface associated with decomposition of sarin on a pristine $\mathrm{ZnO}(10 \overline{10})$ surface based on calculations using PBE functional is shown in Figure S2. Structures of reagent, products and reaction intermediates are collected in Figure S3. Transition state structures are collected in Figure S4.

Our DFT modeling showed that sarin decomposition on a pristine $\mathrm{ZnO}(1 \overline{010})$ surface is a complex process, which can proceed via three main channels: 1) the P-F bond breaking, 2) P$\mathrm{OC}_{3} \mathrm{H}_{7}$ bond breaking, and 3) propene molecule elimination.

We began our study by modeling a decomposition reaction associated with the P-F bond breaking $\left(\boldsymbol{S}_{1}-\boldsymbol{S}_{2}-\boldsymbol{S}_{3}\right)$ in the most stable adsorption Configuration 7 (see Figure 2 of the manuscript). This step requires $123.7 \mathrm{~kJ} \mathrm{~mol}^{-1}$ and proceeds with the energy release of -39.4 $\mathrm{kJ}$ mol $^{-1}$. In configuration $\boldsymbol{S}_{3}$, the fluorine atom remains adsorbed on the surface, whereas a new bond is formed between phosphorus and one of the surface oxygens. Our calculations revealed an alternative mechanism involving the P-F bond breaking $\left(\boldsymbol{S}_{1}-\boldsymbol{S}_{2}-\boldsymbol{S}_{4}-\boldsymbol{S}_{5}-\boldsymbol{S}_{6}\right)$. The first two steps $\left(\boldsymbol{S}_{1}-\boldsymbol{S}_{2}-\boldsymbol{S}_{4}\right)$ of this multistep channel is a transition from the most stable adsorption Configuration 7 to a less favorable Configuration 3 via Configuration 5 (Figure 2 of the manuscript and Figure S1). Our modeling fell short to localize a transition state for the reaction step $\boldsymbol{S}_{2}-\boldsymbol{S}_{4}$. However, we assume that this reaction requires a significantly lower energy than the following steps involving breaking of $\mathrm{P}-\mathrm{OC}_{3} \mathrm{H}_{7}, \mathrm{P}-\mathrm{F}$, and $\mathrm{PO}-\mathrm{C}_{3} \mathrm{H}_{7}$ bonds due to weak bonding between fluorine and zinc atoms. Prior to the P-F bond breaking, a sarin molecule undergoes a conformational transition $\left(\boldsymbol{S}_{4}-\boldsymbol{S}_{5}\right)$ via a rotation of $-\mathrm{OC}_{3} \mathrm{H}_{7}$ fragment about the $\mathrm{P}_{-}-\mathrm{OC}_{3} \mathrm{H}_{7}$ bond by $40^{\circ}$. The calculated barrier of the internal rotation is $10.6 \mathrm{~kJ} \mathrm{~mol}^{-1}$, whereas the structure $\boldsymbol{S}_{5}$ lies $7.2 \mathrm{~kJ} \mathrm{~mol}^{-1}$ higher than $\boldsymbol{S}_{4}$. The P-F bond breaking $\left(\boldsymbol{S}_{5^{-}}\right.$ 
$\boldsymbol{S}_{6}$ ) requires $120.5 \mathrm{~kJ} \mathrm{~mol}^{-1}$. The overall activation barrier of the reaction channel $\boldsymbol{S}_{I^{-}} \boldsymbol{S}_{2}-\boldsymbol{S}_{4}-\boldsymbol{S}_{5}-\boldsymbol{S}_{6}$ is 141.1 and the reaction energy is $-38.2 \mathrm{~kJ} \mathrm{~mol}^{-1}$. Similarly to the configuration $\boldsymbol{S}_{3}$, fluorine atom in the configuration $\boldsymbol{S}_{6}$ remains adsorbed on the surface.

Decomposition of the intermediate configuration $\boldsymbol{S}_{5}$ can also proceed via breaking of P$\mathrm{OC}_{3} \mathrm{H}_{7}$ bond $\left(\boldsymbol{S}_{5}-\boldsymbol{S}_{7}\right)$. This step requires $110.1 \mathrm{~kJ} \mathrm{~mol}^{-1}$. In the structure $\boldsymbol{S}_{7}$, the $\left(-\mathrm{OC}_{3} \mathrm{H}_{7}\right)$ group remains adsorbed on the surface and forms bonds with surface zinc atoms. The overall reaction energy of $\boldsymbol{S}_{1}-\boldsymbol{S}_{2}-\boldsymbol{S}_{4}-\boldsymbol{S}_{5}-\boldsymbol{S}_{7}$ channel is $-17.5 \mathrm{~kJ} \mathrm{~mol}^{-1}$. Our calculations revealed yet another plausible decomposition pathway associated with the $\mathrm{P}-\mathrm{OC}_{3} \mathrm{H}_{7}$ bond breaking $\left(\boldsymbol{S}_{I^{-}} \boldsymbol{S}_{2}-\boldsymbol{S}_{4}-\boldsymbol{S}_{5}-\boldsymbol{S}_{8}-\boldsymbol{S}_{9}\right)$. This mechanism proceeds via an additional conformation transition $\left(\boldsymbol{S}_{5^{-}} \boldsymbol{S}_{\boldsymbol{\delta}}\right)$ corresponding to a rotation of the $-\mathrm{OC}_{3} \mathrm{H}_{7}$ fragment about the $\mathrm{P}-\mathrm{OC}_{3} \mathrm{H}_{7}$ bond by $127.5^{\circ}$. The calculated barrier of the internal rotation $\boldsymbol{S}_{5-} \boldsymbol{S}_{\boldsymbol{\delta}}$ is $14.6 \mathrm{~kJ} \mathrm{~mol}^{-1}$, whereas an intermediate structure $\boldsymbol{S}_{\boldsymbol{8}}$ lies $2 \mathrm{~kJ} \mathrm{~mol}^{-1}$ lower than $\boldsymbol{S}_{5}$. A subsequent breaking of the $\mathrm{P}_{-}-\mathrm{OC}_{3} \mathrm{H}_{7}$ bond $\left(\boldsymbol{S}_{\boldsymbol{g}^{-}} \boldsymbol{S}_{9}\right)$ requires $116.4 \mathrm{~kJ}$ mol-1. The overall reaction energy of the multistep channel $\boldsymbol{S}_{I^{-}} \boldsymbol{S}_{2}-\boldsymbol{S}_{4}-\boldsymbol{S}_{5}-\boldsymbol{S}_{8}-\boldsymbol{S}_{9}$ is $-18.0 \mathrm{~kJ} \mathrm{~mol}^{-1}$.

Our DFT modeling also showed that decomposition of sarin on a pristine $\mathrm{ZnO}(10 \overline{1} 0)$ surface can proceed via an elimination of propene $\left(\boldsymbol{S}_{1}-\boldsymbol{S}_{2}-\boldsymbol{S}_{4}-\boldsymbol{S}_{5}-\boldsymbol{S}_{8}-\boldsymbol{S}_{10}\right.$ and $\left.\boldsymbol{S}_{1}-\boldsymbol{S}_{2}-\boldsymbol{S}_{4} \boldsymbol{S}_{11}\right)$, which involves a concerted breaking of the $\mathrm{PO}-\mathrm{C}_{3} \mathrm{H}_{7}$ and $\mathrm{C}-\mathrm{H}$ bonds. Both channels of propene elimination require lower activation energies than the decomposition channels via the P-F and P$\mathrm{OC}_{3} \mathrm{H}_{7}$ bonds cleavage. Thus, the overall calculated activation barriers of $\boldsymbol{S}_{1}-\boldsymbol{S}_{2}-\boldsymbol{S}_{4}-\boldsymbol{S}_{5}-\boldsymbol{S}_{8}-\boldsymbol{S}_{10}$ and $\boldsymbol{S}_{1}-\boldsymbol{S}_{2}-\boldsymbol{S}_{4}-\boldsymbol{S}_{11}$ channels are 119.8 and $105.7 \mathrm{~kJ} \mathrm{~mol}^{-1}$, respectively. In addition to relatively low activation energies, both mechanisms appeared to be thermodynamically favorable processes. The calculated reaction energies of $\boldsymbol{S}_{1}-\boldsymbol{S}_{2}-\boldsymbol{S}_{4}-\boldsymbol{S}_{5}-\boldsymbol{S}_{8}-\boldsymbol{S}_{10}$ and $\boldsymbol{S}_{1}-\boldsymbol{S}_{2}-\boldsymbol{S}_{4}-\boldsymbol{S}_{11}$ pathways are -77.1 and -48.1 $\mathrm{kJ} \mathrm{mol}^{-1}$. 


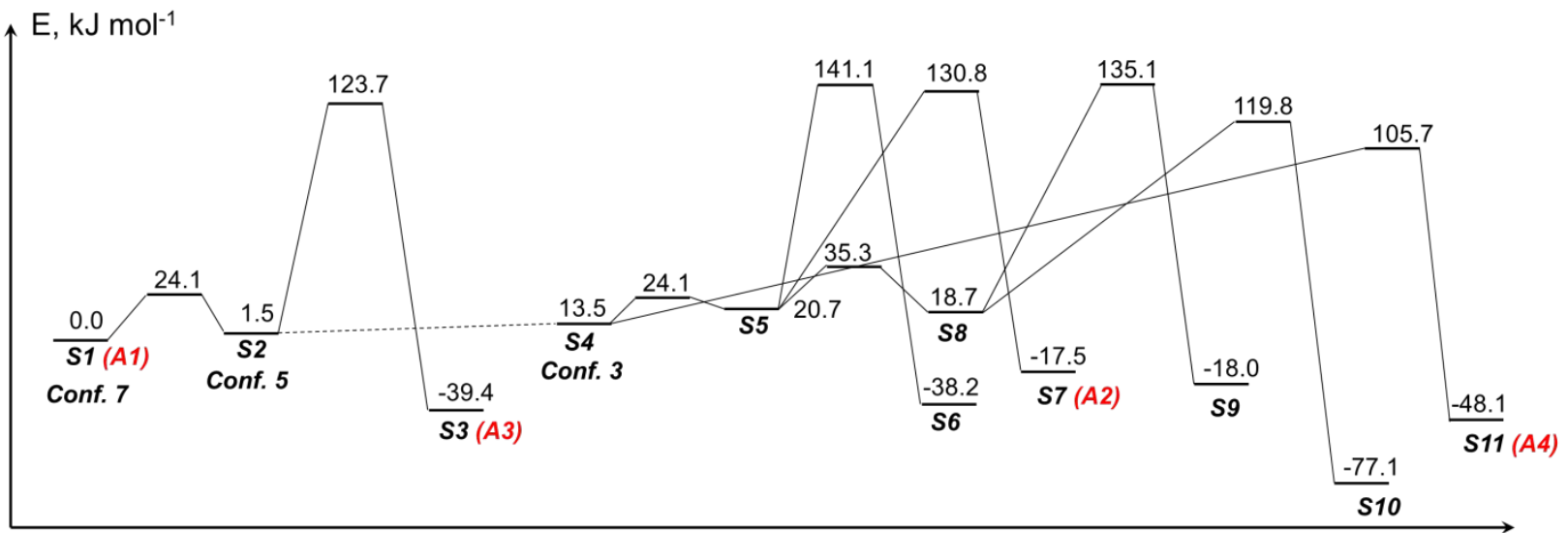

Reaction coordinate

Figure S2 Schematic representation of the potential energy surface associated with decomposition of sarin on a pristine $\mathrm{ZnO}(10 \overline{1} 0)$ surface. Activation barriers and reaction energies are calculated relative to the structure $\mathbf{A}_{1}$ using PBE functional.
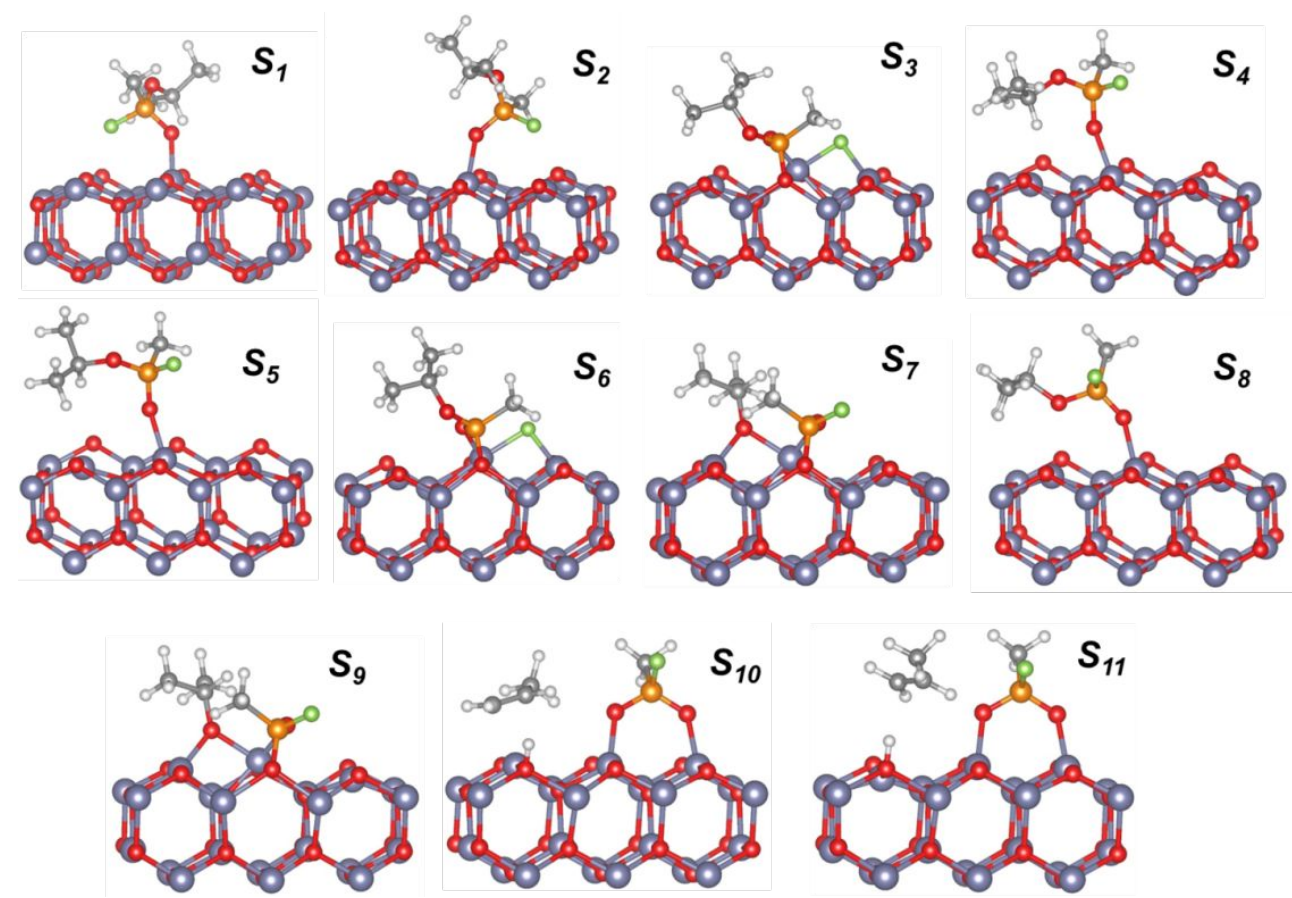

Figure S3 Structures of reagent, products and intermediates involved in sarin decomposition on a pristine $\mathrm{ZnO}(10 \overline{1} 0)$ surface. 


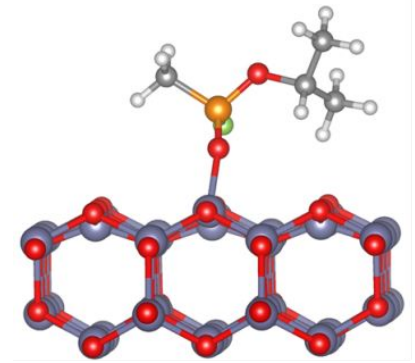

$T S S_{1}-S_{2}$

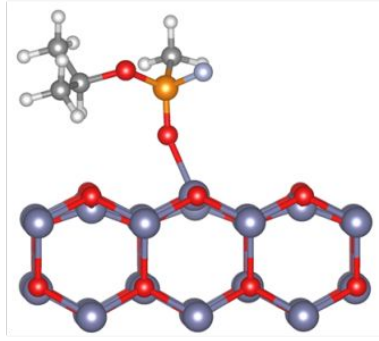

$T S S_{4}-S_{5}$

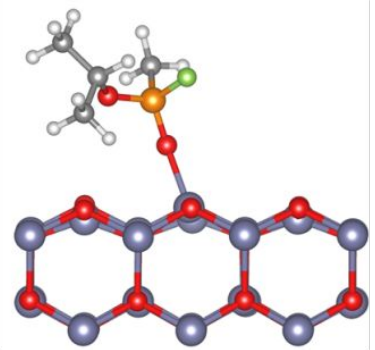

$T S S_{5}-S_{8}$

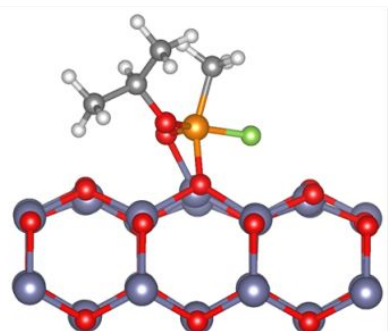

$T S S_{2}-S_{3}$

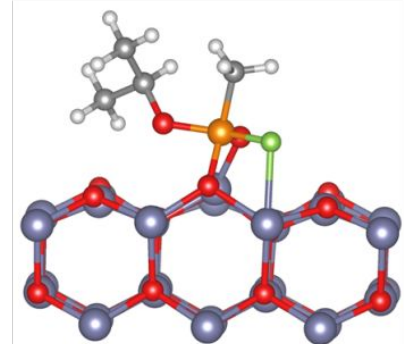

$T S S_{5}-S_{6}$

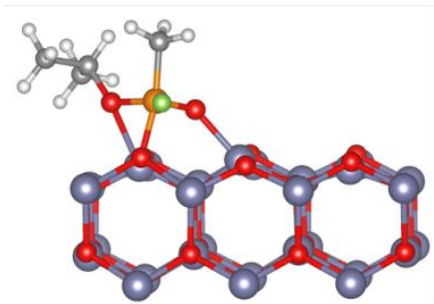

$T S S_{8}-S_{9}$

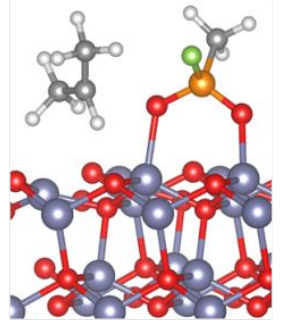

$T S S_{4}-S_{11}$

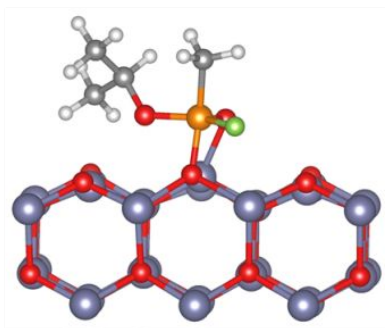

$T S S_{5}-S_{7}$

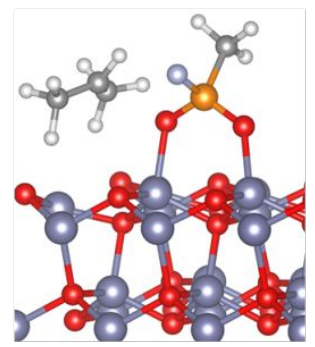

$T S S_{8}-S_{10}$

Figure S4 Transition state structures of reactions corresponding to sarin decomposition on a pristine $\mathrm{ZnO}(10 \overline{1} 0)$ surface. 


\section{Mechanisms of sarin decomposition on a hydroxylated $\mathrm{ZnO}(10 \overline{10})$ surface (results obtained using PBE functional)}

A schematic representation of the potential energy surface associated with decomposition of sarin on a hydroxylated $\mathrm{ZnO}(10 \overline{1} 0)$ surface based on calculations using PBE functional is depicted in Figure S5. The structures of the reagent, products and reaction intermediates are collected in Figure S6. Transition state structures are collected in Figure S7.

In modeling sarin decomposition on a hydroxylated $\mathrm{ZnO}$ surface, we used the most favorable adsorption Configuration 10 (see Figure 3 of the manuscript). Our calculations show that the P-F bond breaking and the IMPA molecule formation is the main mechanism of sarin hydrolysis on $\mathrm{ZnO}$. We found two plausible mechanisms for this reaction. The first mechanism $\left(\mathrm{B}_{1}-\mathrm{B}_{2}\right)$ is a one step process that requires $145.1 \mathrm{~kJ} \mathrm{~mol}^{-1}$ and proceeds with an energy release of $40.9 \mathrm{~kJ} \mathrm{~mol}{ }^{-1}$. The alternative mechanism $\mathrm{B}_{1}-\mathrm{B}_{3}-\mathrm{B}_{4}$ is a stepwise process. The first step $\left(\mathrm{B}_{1}-\mathrm{B}_{3}\right)$ involves a rotation of the $-\mathrm{OC}_{3} \mathrm{H}_{7}$ group about the $\mathrm{P}-\mathrm{O}$ bond by $\sim 36^{\circ}$ (from $32.3^{\circ}$ in $\mathrm{B}_{1}$ to $68.6^{\circ}$ in $B_{3}$ ). Such a rotation requires $37.9 \mathrm{~kJ} \mathrm{~mol}^{-1}$, whereas the structure $\mathrm{B}_{3}$ lies $20.2 \mathrm{~kJ} \mathrm{~mol}^{-1}$ higher than $\mathrm{B}_{1}$. The subsequent hydrolysis of the P-F bond $\left(\mathrm{B}_{3}-\mathrm{B}_{4}\right)$ requires an additional $71.8 \mathrm{~kJ} \mathrm{~mol}^{-1}$. The IMPA molecule can further adsorb on a $\mathrm{Zn}$ atom $\left(\mathrm{B}_{4}-\mathrm{B}_{5}\right)$ through a hydrogen shift to one of the surface water molecules. This exothermic step has a $11.9 \mathrm{~kJ} \mathrm{~mol}^{-1}$ activation barrier. The structure $\mathrm{B}_{5}$ lies $7.1 \mathrm{~kJ}$ mol-1 lower than $\mathrm{B}_{4}$. The next step $\left(\mathrm{B}_{5}-\mathrm{B}_{6}\right)$ involves a migration of the fluorine atom between neighboring $\mathrm{Zn}$ atoms accompanied by desorption of a water molecule. This step requires only $2.3 \mathrm{~kJ} \mathrm{~mol}^{-1}$ and has the reaction energy of $-8.2 \mathrm{~kJ} \mathrm{~mol}^{-1}$. The calculated overall activation energy of the stepwise process $B_{1}-B_{6}$ is 92.0 and the reaction energy is $-52.7 \mathrm{~kJ} \mathrm{~mol}^{-1}$. Remarkably, a fluorine atom remains on the surface rather than leaving as a hydrogen fluoride molecule, while the IMPA molecule also remains adsorbed on the surface atop a $\mathrm{Zn}$ atom. A considerably high 
energy $\left(259.0 \mathrm{~kJ} \mathrm{~mol}^{-1}\right)$ is required to remove the IMPA molecule and fluorine atom in the form of HF molecule from the surface. This suggests that both fluorine atom and IMPA molecule will remain on the surface after the sarin decomposition.

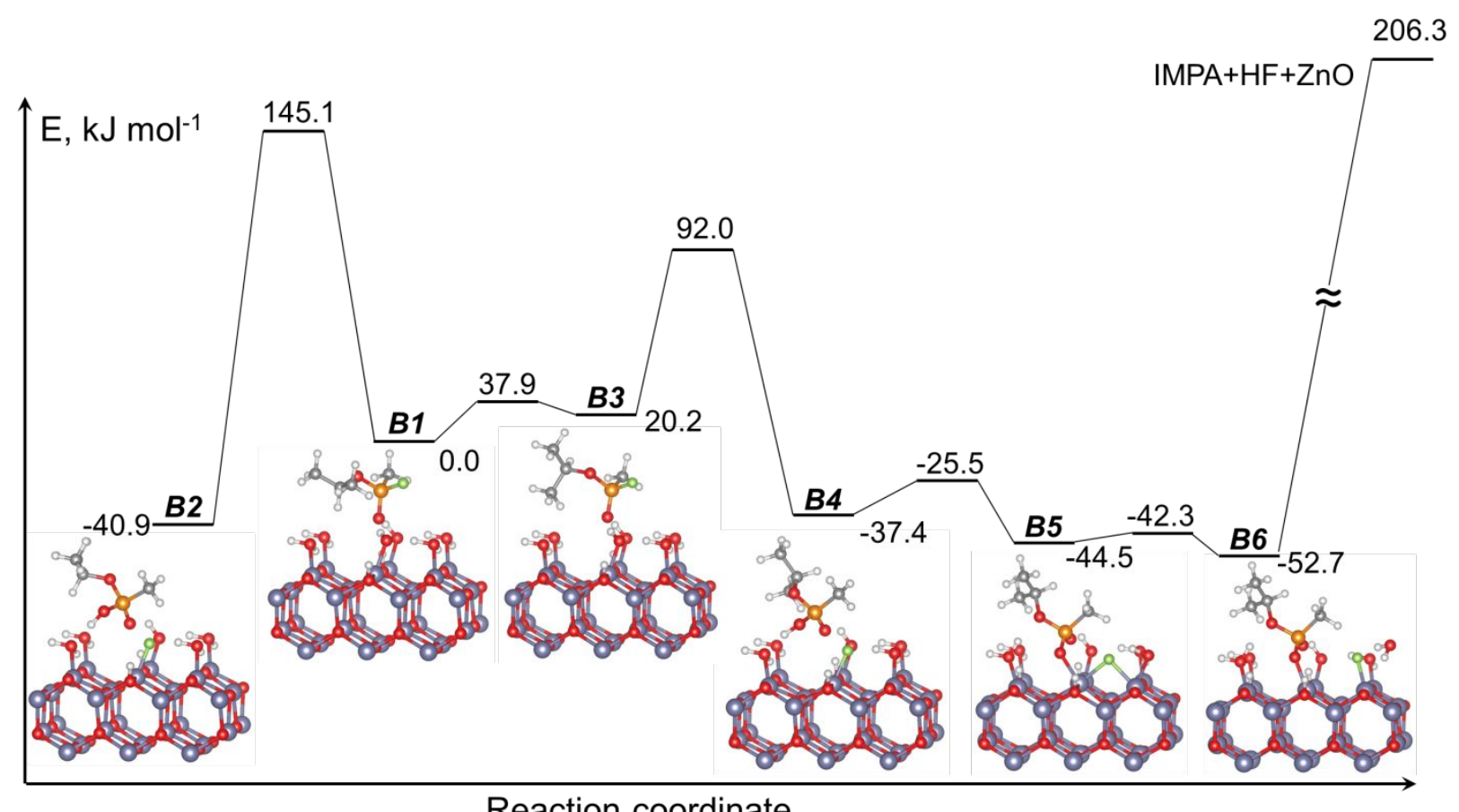

Figure S5. Schematic representation of the potential energy surface associated with decomposition of sarin on hydroxylated $\mathrm{ZnO}(10 \overline{1} 0)$. Activation barriers and reaction energies are calculated relative to $\mathrm{B}_{1}$ state using a $\mathrm{PBE}$ functional. 


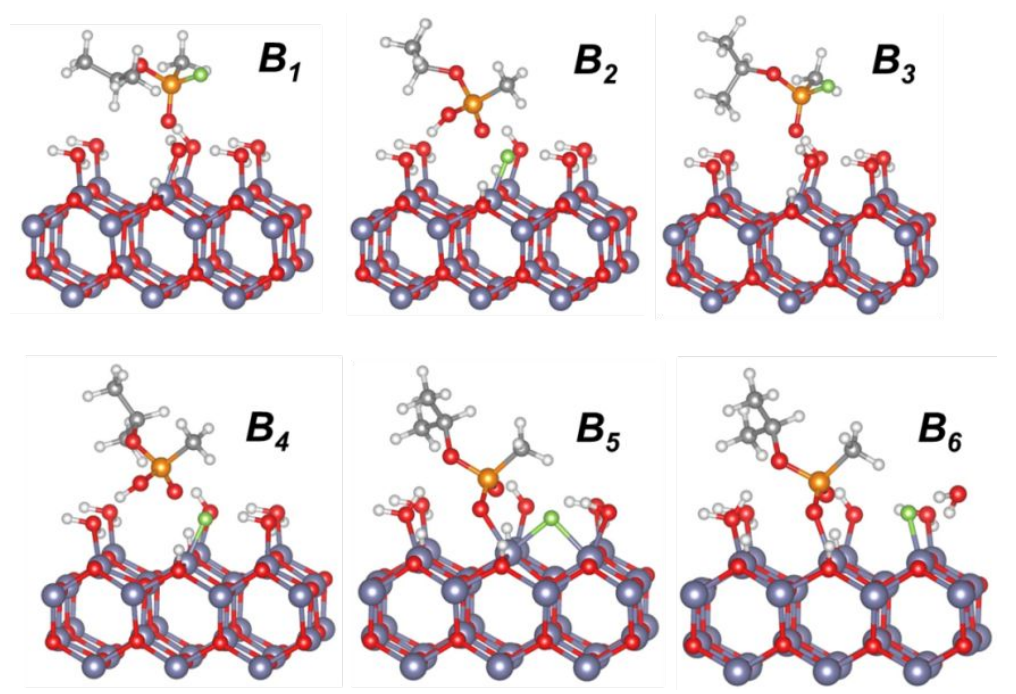

Figure S6. Structures of the reagent, products and reaction intermediates involved in sarin decomposition on a pristine $\mathrm{ZnO}(10 \overline{1} 0)$ surface.

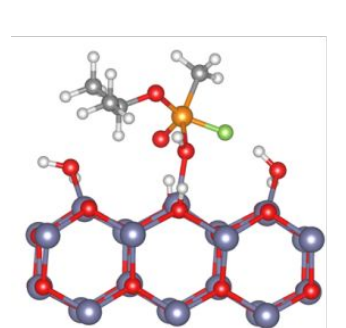

TS $B_{1}-B_{2}$

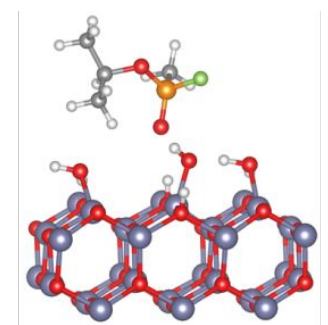

TS $B_{1}-B_{3}$

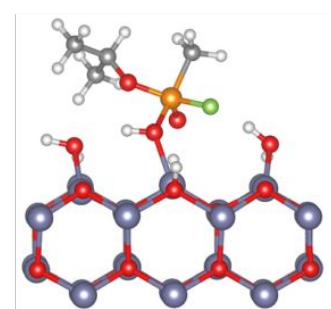

$T S B_{3}-B_{4}$

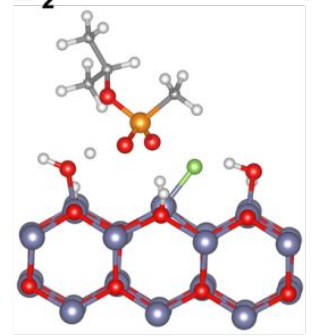

$T S B_{4}-B_{5}$

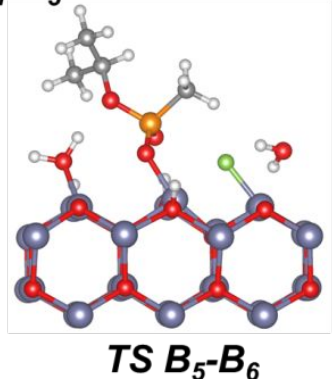

Figure S7 Transition state structure of reactions corresponding to sarin decomposition on a pristine $\mathrm{ZnO}(10 \overline{1} 0)$ surface. 


\section{Mechanisms of sarin decomposition on a hydroxylated $\mathrm{ZnO}$ (1010) surface via water displacement (results obtained using PBE functional)}

Schematic representation of the potential energy surface associated with decomposition of sarin on a hydroxylated $\mathrm{ZnO}(10 \overline{1} 0)$ surface via a water displacement path is depicted in Figure S8 based on calculations using PBE functional. The structures of the reagent, products and reaction intermediates are collected in Figure S9. Transition state structures are collected in Figure S10.

The most favorable adsorption Configuration 10 (see Figure 3 of the manuscript) served as an initial state reaction pathways involving a displacement of one of the intact water molecules on $\mathrm{ZnO}(10 \overline{1} 0)$ surface that has been covered with a water monolayer. Our calculations show that the displacement of the water molecule $\left(\mathrm{B}_{1}-\mathrm{C}_{1}\right)$ is an endothermic process, which requires $91.9 \mathrm{~kJ}$ mol $^{-1}$. The $\mathrm{C}_{1}$ structure lies $73.3 \mathrm{~kJ} \mathrm{~mol}^{-1}$ above $\mathrm{B}_{1}$. The subsequent decomposition of $\mathrm{C}_{1}$ can proceed via several pathways. The first channel $\mathrm{C}_{1}-\mathrm{C}_{2}-\mathrm{C}_{3}$ yields a $\mathrm{HF}$ molecule viaa nucleophilic addition to sarin of the water molecule displaced during the previous step $\left(\mathrm{C}_{1}-\mathrm{C}_{2}\right)$ and the formation of an intermediate structure $\mathrm{C}_{2}$ with a penta-coordinated phosphorus atom. The reaction step $\mathrm{C}_{1}-\mathrm{C}_{2}$ requires $60.7 \mathrm{~kJ} \mathrm{~mol}^{-1}$, whereas $\mathrm{C}_{2}$ lies $28.5 \mathrm{~kJ} \mathrm{~mol}^{-1}$ higher than $\mathrm{C} 1$. The subsequent elimination of $\mathrm{HF}\left(\mathrm{C}_{2}-\mathrm{C}_{3}\right)$ requires only $2.7 \mathrm{~kJ} \mathrm{~mol}^{-1}$. The overall calculated activation barrier and reaction energy of reaction pathway $\mathrm{B}_{1}-\mathrm{C}_{1}-\mathrm{C}_{2}-\mathrm{C}_{3}$ are, 134.0 and $-15.9 \mathrm{~kJ}$ mol-1, respectively.

Alternatively, decomposition of $\mathrm{C}_{1}$ can proceed via an elimination of the iso-propanol molecule $\left(\mathrm{C}_{1}-\mathrm{C}_{4}-\mathrm{C}_{5}\right)$ or the P-F bond fission $\left(\mathrm{C}_{1}-\mathrm{C}_{4}-\mathrm{C}_{6}\right)$, which, in turn, yields the IMPA molecule and $\mathrm{F}$ atom bound to $\mathrm{Zn}$ atom. Both of these reaction channels have a common reaction step $\mathrm{C}_{1^{-}}$ $\mathrm{C}_{4}$, involving the formation of an intermediate structure with a penta-coordinated phosphorus atom via the nucleophilic addition of surface hydroxyl. This step requires $66.1 \mathrm{~kJ} \mathrm{~mol}^{-1}$. The pentacoordinated intermediate $\mathrm{C}_{4}$ lies $47.3 \mathrm{~kJ} \mathrm{~mol}^{-1}$ above $\mathrm{C}_{1}$. An elimination of iso-propanol $\left(\mathrm{C}_{4}-\mathrm{C}_{5}\right)$ 
has the activation barrier of $32.6 \mathrm{~kJ} \mathrm{~mol}^{-1}$, which gives the overall activation barrier of $153.2 \mathrm{~kJ}$ mol $^{-1}$ for the $\mathrm{B}_{1}-\mathrm{C}_{1}-\mathrm{C}_{4}-\mathrm{C}_{5}$ process. The elimination of iso-propanol is a slightly exothermic process with the overall reaction energy of $-3.2 \mathrm{~kJ} \mathrm{~mol}^{-1}$. The formation of the IMPA molecule through P-F bond fission in $\mathrm{C}_{4}$ is a more favorable process with the calculated activation barrier of $10.4 \mathrm{~kJ}$ $\mathrm{mol}^{-1}$. Hence, the formation of the penta-coordinated intermediate structure $\mathrm{C}_{4}$ from $\mathrm{C}_{1}$ is the ratelimiting step of the stepwise process $\mathrm{B}_{1}-\mathrm{C}_{1}-\mathrm{C}_{4}-\mathrm{C}_{6}$ with the overall activation barrier of $131.0 \mathrm{~kJ}$ mol-1. The reaction pathway $\mathrm{B}_{1}-\mathrm{C}_{1}-\mathrm{C}_{4}-\mathrm{C}_{6}$ is a thermodynamically favorable process and proceeds with $37.5 \mathrm{~kJ} \mathrm{~mol}^{-1}$ energy release.

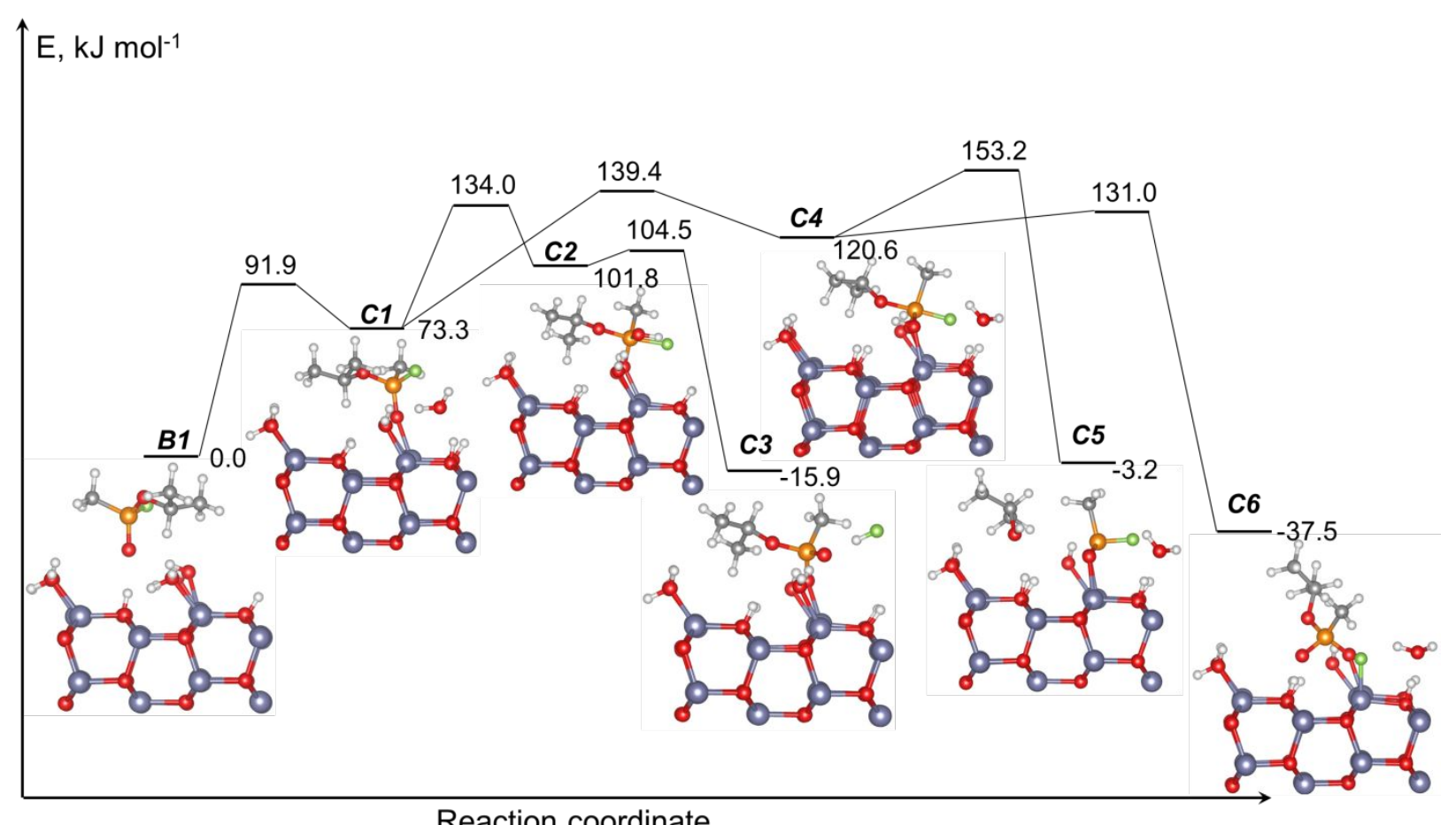

Figure S8. Schematic representation of the potential energy surface associated with decomposition of sarin on hydroxylated $\mathrm{ZnO}(10 \overline{1} 0)$ via the water displacement reaction. Activation barriers and reaction energies are calculated relative to $\mathrm{B}_{1}$ state using a PBE functional. 

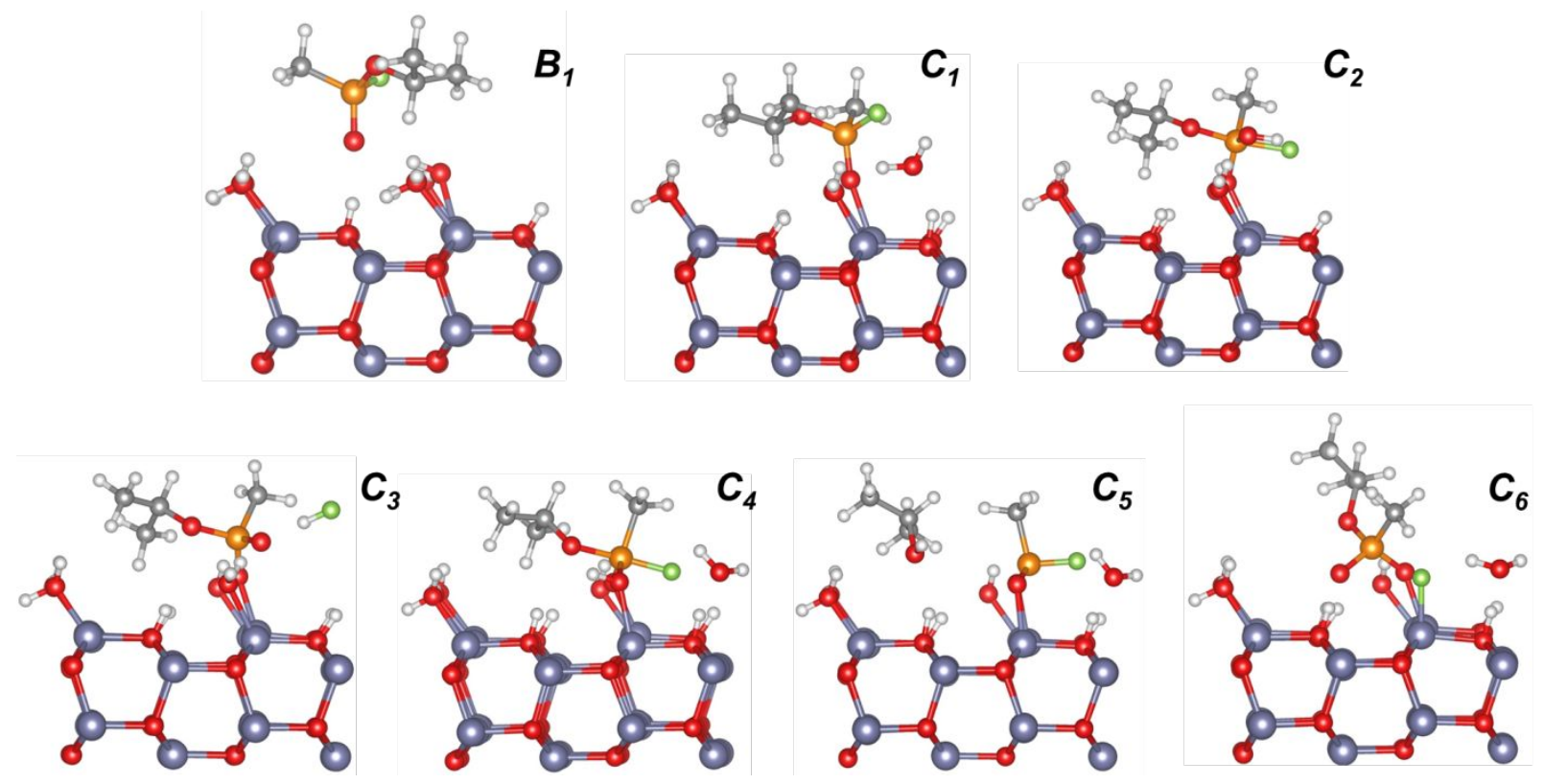

Figure S9. Structures of the reagent, products and reaction intermediates involved in sarin decomposition on a pristine $\mathrm{ZnO}(10 \overline{1} 0)$ surface via water displacement.

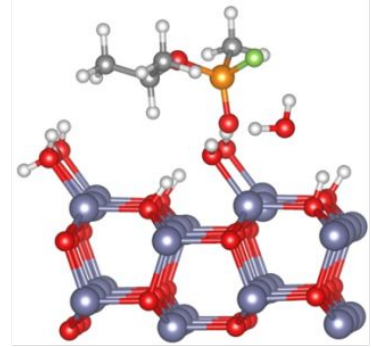

TS $B_{1}-C_{1}$

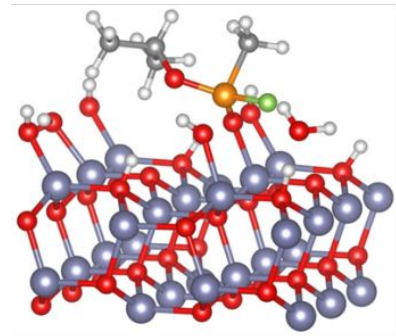

TS $\mathrm{C}_{1}-\mathrm{C}_{4}$

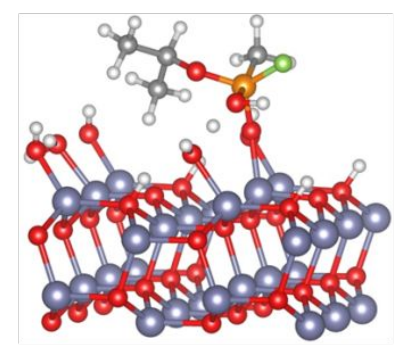

TS $C_{1}-C_{2}$

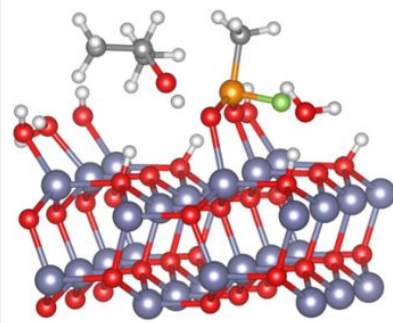

$\mathrm{TS}_{4}-\mathrm{C}_{5}$

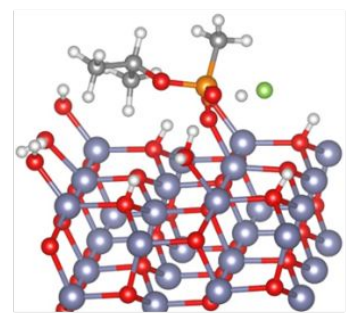

TS $\mathrm{C}_{2}-\mathrm{C}_{3}$

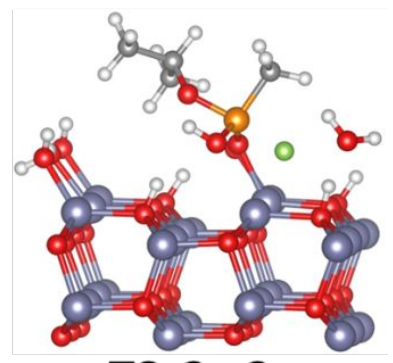

$T S C_{5}-C_{6}$

Figure S10 Transition state structure of reactions corresponding to sarin decomposition on a pristine $\mathrm{ZnO}(10 \overline{1} 0)$ surface via water displacement. 


\section{Calculations of the pre-exponential factor for the methanol elimination reaction}

The pre-exponential factor for reactions with a well-defined transition state can be calculated by applying the transition state theory (TST) $)^{3}$ using an expression:

$$
k_{T S T}=A_{T S T} e^{-\Delta E / K_{b} T}=\frac{K_{b} T}{h} \frac{q_{v i b}^{\#} q_{\text {rot }}^{\#} q_{\text {trans }}^{\#}}{q_{\text {vib }} q_{\text {rot }} q_{\text {trans }}} e^{-\Delta E / K_{b} T}
$$

where $A_{T S T}$ is pre-exponential factor, $K_{\mathrm{b}}$ is Boltzmann constant, $h$ is Planck constant, $\mathrm{T}$ is

temperature, $q_{v i b}^{\#}, q_{\text {rot }}^{\#}$, and $q_{\text {trans }}^{\#}$ are vibrational, rotational and translational partition functions of the transition state, $q_{v i b}, q_{\text {rot }}$, and $q_{\text {trans }}$ are vibrational, rotational and translational partition functions of the reagent, $\Delta \mathrm{E}$ is the activation barrier calculated as the energy difference between the total energies of the compound at the transition $\left(E^{\#}\right)$ state and the reagent $(E)$ :

$$
\Delta E=E^{\#}-E
$$

In calculating the pre-exponential factor for the methanol elimination reaction, we used a simplified version of equation 1 taking into consideration that translational and rotational partition functions of transition state are close to those of the reactant.

$$
A_{T S T}=\frac{K_{b} T q_{v i b}^{\#}}{h q_{v i b}}
$$

